\title{
Estimation of Genetic Variability of Nodulation Characters and their Association with Different Agromorphic Characters and Yield in Chickpea (C. arietinum L.)
}

\author{
L. Priyadarsini ${ }^{1}$, Prabhat K. Singh ${ }^{2 *}$, C. Chatterjee ${ }^{1}$, R. Sadhukhan ${ }^{1}$ and T. Biswas ${ }^{3}$ \\ ${ }^{1}$ Department of Genetics and Plant Breeding, Bidhan Chandra Krishi Viswavidyalaya, \\ Mohanpur-741252, Nadia, West Bengal, India \\ ${ }^{2}$ Department of Genetics and Plant Breeding, M. S. Swaminathan School of Agriculture, CUTM, \\ Paralekhmundhi, Odisha-761211, India \\ ${ }^{3}$ Department of Agricultural Chemistry and Soil Science, Bidhan Chandra Krishi \\ Viswavidyalaya, Mohanpur-741252, Nadia, West Bengal, India \\ *Corresponding author
}

\begin{abstract}
Keywords
Chickpea,

Correlation,

Nodulation

characters, Path

analysis, Root

morphology. agromorphic traits including yield. High GCV and PCV for nodule number per plant and seed yield per plant were estimated. High heritability with high GA\% over mean was noticed in case of nodule number per plant, nodule dry weight per plant, number of pods per plant, 100 pod weight, 100 seed weight, harvest index and seed yield per plant. Correlation studies revealed that four characters viz., primary root number per plant, root length, root dry weight and shoot length were significantly positively correlated with yield (both at genotypic and phenotypic level). While, nodule dry weight per plant, number of pods per plant, 100 pod weight and harvest index were positively correlated with seed yield per plant at genotypic and phenotypic level. Path analysis exhibited that root dry weight, root length, shoot length, nodule dry weight, days to $50 \%$ flowering, plant height, number of secondary branches, number of pods per plant, number of seeds per pod and harvest index had direct positive effect on seed yield per plant. Among the desi types BG 256, Pusa 362, PDG 3, GNG 146 and CSJD 884 had more nodule dry weight per plant and seed yield per plant. Among kabuli types HK 1 and HK 2 had higher nodule weight but lower seed yield as compared to L 550. In case of majority of varieties, desi types had better nodulating behaviour including nodule number, nodule dry weight and better economic yield potential in contrast to kabuli types. Overall the variety BG 256 and Pusa 362 appeared to be very good performer at New Alluvial Zone.
\end{abstract}

A B S T R A C T

The present investigation was under taken during rabi 2014-15 under rice fallow condition at District Seed Farm Kalyani, BCKV, W.B. Fifteen elite genotypes of chickpea (3 kabuli and 12 desi) were taken to evaluate and select the better yielding genotype(s) based on the nodulation behavior as well as root morphology, suitable for new alluvial zone of West Bengal and also to estimate the correlation among different underground and above ground

\section{Introduction}

Chickpea ( $C$. arietinum L.) is considered now as the third most important pulse in the world and is widely cultivated in subtropical and warm temperate region (Mansfeld, 2008). 
Chickpea belongs to the family Fabaceae and sub family Papilionaceae. There are mainly two kinds of chickpea commercially cultivated i.e. desi type (microsperma race) having small, darker seeds and rough is mostly cultivated in Indian subcontinent, Ethiopia, Mexico and Iran (Mansfeld, 2008). Kabuli type (macrosperma race) having lighter coloured, smoother seed coat and large seeds generally grown in Europe, Northen Africa, Afganistan and also introduce to Indian subcontinent. The race microsperma believe to derive from microsperma (Moreno and Cubero, 1978). Like other pulses it fixes atmospheric nitrogen and enhances soil fertility as well as saving the fertilizer cost in subsequent crops due to residual nitrogen. It is an excellent source of protein, fiber, complex carbohydrate, vitamin, minerals. This crop is a rich source of protein, vitamin $A$ and iron (Bender and Bender, 2005). Absence of suitable strains, small population size and poor survival of Rhizobia cause problems in nodules formation (Kantar et al., 2007). Grain yield of chickpea increased considerably with rhizobial application (Khattak et al., 2006 and Romdhane et al., 2008). The productivity and production of chickpea have been shown to be increasing from 2007 (845 kg/ha) to $920 \mathrm{~kg} / \mathrm{ha}$ in 2013 and production was increased from 6.33 million tons in 2007 to 8.83 million tons in 2013 (FAO, 2013). The objective of the present study was to evaluate and select the better yielding chickpea genotype(s) both in kabuli and desi type in the new alluvial zone of West Bengal based on the nodulation behavior as well as root morphology and to estimate the correlation among different underground and above ground agromorphic traits including yield.

\section{Materials and Methods}

Materials consisted of three varieties of macrosperma (kabuli) and twelve varieties of microsperma (desi) group having different breeding origin and were grown in RBD with three replications at the District Seed Farm, located in the Gangetic New Alluvial Zone of West Bengal having latitude of $22^{\circ} 57^{\prime} \mathrm{N}$ and longitude of $88^{\circ} 20^{\prime} \mathrm{E}$. The experimental plot was new alluvial soil type with good drainage facilities with sandy loam texture. It had $0.51 \%$ coarse sand (international pipette method (pipper 1950) with $\mathrm{pH}$ of 6.9. The date of sowing was $23^{\text {rd }}$ November 2014 . The study of underground characters such as nodule number per plant, nodule dry weight per plant, primary root number, root length, root dry weight was taken into consideration at 75 days of growth stage. Similarly, above ground characters such as shoot length and shoot dry biomass were also studied at the same age. Standard methodology was applied and observations were recorded from 10 plants of each replication for each variety. The yield attributing characters such as days to $50 \%$ flowering, days to harvesting, plant height (at the time of harvesting), number of primary branches, number of secondary branches, number of pods per plant, 100 pod weight, number of seeds per pod, 100 seed weight, harvest index, seed yield per plant was observed at the time of harvest.

\section{Results and Discussion}

The analysis of variance with respect to the eighteen traits is presented in table 1 . The mean sum of square due to all genotypes for all the above-mentioned characters were observed to be highly significant which indicated significant differences among the experimental materials indicating a definite scope for improving the characters through further selection. Different variability parameters viz., mean, range, phenotypic variance, genotypic variance, environmental variance, coefficient of variation (CV), genotypic coefficient of variation (GCV), phenotypic coefficient of variation (PCV), 
heritability (BS), genetic advance (GA) and genetic advance as a percentage over mean of 15 genotypes are represented in table 2 . Genotypic and phenotypic correlation coefficients are presented in table 3 and path analysis in table 4. In general, phenotypic variance was higher than corresponding genotypic variance against all the characters which indicated the presence of environmental influence on these characters. The highest estimates of phenotypic and genotypic variance were observed in nodule dry weight (mg) which was followed by number of pods per plant and harvest index. The results also highlighted that nodule dry weight per plant, root length, 100 seed weight and harvest index had less environmental influences compared to other traits. These findings were in agreement with that of Ramgiry et al., (1997) in faba bean and Borate et al., (2010) in chickpea. These findings corroborated earlier works of different workers for one or more characters like Khan et al., (2006) for plant height, days to maturity, days to $50 \%$ flowering in gram and Ramgiry et al., (1997) for seed yield per plant in faba bean. High heritability was observed for most of the characters studied viz. 100 seed weight, shoot length, harvest index, nodule dry weight per plant, primary root number per plant, root length and nodule number per plant respectively. Among these characters plant height, number of primary branch, number of secondary branch, shoot dry biomass and number of seed per plant recorded lower heritability. These findings were in conformity with different workers for one or more characters e.g. Singh et al., (1995) for nodule dry weight and nodule number per plant in soybean and Rana et al., (1995) for 100 seed weight in chickpea. Genetic advance as percentage over mean was highest for nodule number per plant $(98.41 \%)$ followed by seed yield per plant $(78.95 \%)$ and 100 seed weight $(65.13 \%)$ respectively. The lowest GA as percentage over mean was observed in plant height $(10.88 \%)$ followed by days to maturity $(14.31 \%)$ and days to $50 \%$ flowering $(17.43 \%)$. Pods per plant had also high GA as \% over mean and this finding was corroboration with Patel et al., (2008) in moth bean. High heritability with high GA as percent over mean was obtained for nodule dry weight plant, seed yield per plant and 100 seed weight. These findings were in agreement with that of Ramgiry et al., (1997) for 100 seed weight and seed yield per plant in faba bean. It indicated the predominance of additive gene action for controlling these characters. Therefore, these characters can be selected directly. High heritability with low GA as percent over mean was observed in shoot length and root dry weight. It suggested non-additive gene action for the expressions of these characters.

The genotypic and phenotypic correlation coefficients are presented in table 3. Correlation studies revealed that all the four characters i.e. primary root number per plant, root length, root dry weight, shoot length were significantly positively correlated with yield (both at genotypic and phenotypic level) at 75 days growth stage (flowering stage). Nodule dry weight per plant was positively correlated with shoot dry biomass both at genotypic and phenotypic level. Nodule dry weight per plant, number of pods per plant, 100 pod weight and harvest index were positively correlated with seed yield per plant at genotypic and phenotypic level. Residual effect of path coefficient analysis was low (0.289). It indicated that the number of characters, chosen for the study was very much appropriate for yield determination in the present study. Root dry weight, harvest index, root length, number of seed per pod, shoot length, number of pods per plant, nodule dry weight per plant, days to $50 \%$ flowering, plant height and number of secondary branch had direct positive effect on seed yield per plant respectively. 
Table.1 Analysis of variance of various characters in 15 chickpea genotypes

\begin{tabular}{|l|l|c|c|c|}
\hline \multicolumn{1}{|c|}{ Characters } & \multicolumn{1}{|c|}{$\begin{array}{c}\text { Source of Variation at different d.f } \\
\text { Replication (2) }\end{array}$} & Genotypes (14) & Error (28) \\
\hline 1 & & 0.32 & $12.2^{* *}$ & 0.15 \\
\hline 2 & Nodule number per plant & 3.37 & $74.0^{* *}$ & 54.02 \\
\hline 3 & Nodule dry weight per plant (mg) & 0.49 & $48.3^{* *}$ & 0.40 \\
\hline 4 & Primary root no. per plant & 0.37 & $27.4^{* *}$ & 0.24 \\
\hline 5 & Root length (cm) & 0.00 & $0.1^{* *}$ & 0.00 \\
\hline 6 & Root dry weight (g) & 0.65 & $40.6^{* *}$ & 0.27 \\
\hline 7 & Shoot length (cm) & 0.08 & $1.0^{* *}$ & 0.11 \\
\hline 8 & Shoot dry biomass (g) & 20.27 & 5.91 \\
\hline 9 & Days to 50\% flowering & 2.75 & $136.0^{* *}$ & 2.49 \\
\hline 10 & Days to maturity & 5.94 & $1911^{* *}$ & 9.46 \\
\hline 11 & Plant height (cm) & 0.15 & $63.8^{* *}$ & 0.12 \\
\hline 12 & Number of primary branches & 0.01 & $0.9^{* *}$ & 1.62 \\
\hline 13 & Number of secondary branches & 1.33 & $12.6^{* *}$ & 10.35 \\
\hline 14 & No. of Pods per Plant & 3.27 & $544.9^{* *}$ & 6.50 \\
\hline 15 & 100 Pod Weight (g) & 0.05 & $183.8^{* *}$ & $0.2^{* *}$ \\
\hline 16 & No of Seeds per Pod & 1.36 & $92.8^{* *}$ & 0.02 \\
\hline 17 & 100 Seed Weight (g) & 1.17 & $221.0^{* *}$ & 1.67 \\
\hline 18 & Harvest index & 1.89 & $86.6^{* *}$ & 1.39 \\
\hline
\end{tabular}

Table.2 Variability parameters of chick pea for various characters

\begin{tabular}{|c|c|c|c|c|c|c|c|c|c|c|c|c|}
\hline \multirow{2}{*}{$\begin{array}{l}\text { Sl. } \\
\text { No. }\end{array}$} & \multirow{2}{*}{ characters } & \multirow{2}{*}{$\begin{array}{l}\text { Grand } \\
\text { mean }\end{array}$} & \multirow[t]{2}{*}{ Range } & \multicolumn{3}{|c|}{ Variance } & \multirow[t]{2}{*}{$\mathrm{CV}$} & \multirow[t]{2}{*}{ GCV } & \multirow[t]{2}{*}{ PCV } & \multirow[t]{2}{*}{$h^{2}(b s)$} & \multirow[t]{2}{*}{ GA } & \multirow{2}{*}{$\begin{array}{c}\text { GA\% over } \\
\text { Mean }\end{array}$} \\
\hline & & & & Phenotypic & Genotypic & Environment & & & & & & \\
\hline 1 & Nodule no & 4.13 & $1.8-9.8$ & 4.18 & 4.03 & 0.15 & 4.16 & 48.65 & 49.55 & 0.96 & 4.04 & 98.41 \\
\hline 2 & Nodule dry Weight(mg) & 198.00 & $111.4-292.6$ & 2394.03 & 2340.01 & 54.02 & 1.89 & 24.42 & 24.70 & 0.98 & 98.04 & 49.73 \\
\hline 3 & Primary root no. & 23.76 & $15.9-31.5$ & 16.35 & 15.96 & 0.40 & 1.27 & 16.81 & 17.02 & 0.98 & 8.09 & 34.22 \\
\hline 4 & Root length $(\mathrm{cm})$ & 22.51 & $18.6-31.1$ & 9.28 & 9.04 & 0.24 & 1.05 & 13.35 & 13.53 & 0.97 & 6.08 & 27.15 \\
\hline 5 & Root dry weight (g) & 0.39 & $0.2-0.6$ & 0.02 & 0.02 & 0.00 & 1.82 & 33.38 & 35.78 & 0.87 & 0.25 & 64.13 \\
\hline 6 & Shoot length $(\mathrm{cm})$ & 24.08 & $19.1-30.9$ & 13.71 & 13.44 & 0.27 & 1.02 & 15.23 & 15.38 & 0.98 & 7.44 & 31.06 \\
\hline 7 & Shoot dry biomass (g) & 1.92 & $1.0-3.2$ & 0.38 & 0.27 & 0.11 & 5.46 & 27.18 & 32.18 & 0.71 & 0.90 & 47.30 \\
\hline 8 & Days to $50 \%$ flowering & 73.00 & $64.6-91.3$ & 49.27 & 43.36 & 5.91 & 1.66 & 9.02 & 9.62 & 0.88 & 12.66 & 17.43 \\
\hline 9 & Days to maturity & 112.18 & $100.6-127.0$ & 65.64 & 63.15 & 2.49 & 0.70 & 7.08 & 7.22 & 0.96 & 15.98 & 14.31 \\
\hline 10 & Plant height $(\mathrm{cm})$ & 65.30 & $58.7-75.4$ & 27.56 & 18.10 & 9.46 & 2.33 & 6.52 & 8.04 & 0.66 & 7.07 & 10.88 \\
\hline 11 & No. of primary Branches & 2.55 & $2.0-3.8$ & 0.37 & 0.24 & 0.12 & 4.99 & 19.41 & 23.78 & 0.67 & 0.83 & 32.64 \\
\hline 12 & No. of secondary Branches & 10.12 & $7.3-15.2$ & 5.28 & 3.66 & 1.62 & 5.60 & 18.90 & 22.70 & 0.69 & 3.27 & 32.43 \\
\hline 13 & No. of Pods/Plant & 50.63 & $33.4-74.3$ & 188.52 & 178.17 & 10.35 & 3.05 & 26.36 & 27.12 & 0.95 & 26.60 & 52.79 \\
\hline 14 & 100 Pod Weight(g) & 32.29 & $22.25-45.76$ & 65.60 & 59.11 & 6.50 & 3.66 & 23.81 & 25.08 & 0.90 & 14.96 & 46.56 \\
\hline 15 & No of Seeds/Pod & 1.56 & $1.2-2.1$ & 0.09 & 0.07 & 0.02 & 2.83 & 16.71 & 19.04 & 0.77 & 0.47 & 30.22 \\
\hline 16 & 100 Seed Weight $(\mathrm{g})$ & 17.49 & $10.077-29.073$ & 31.10 & 30.83 & 0.27 & 1.46 & 31.76 & 31.89 & 0.99 & 11.33 & 65.13 \\
\hline 17 & $\mathrm{HI}$ & 36.28 & $23.49-52.157$ & 74.80 & 73.12 & 1.67 & 1.88 & 23.57 & 23.84 & 0.98 & 17.33 & 48.01 \\
\hline 18 & Seed Yield/Plant (g) & 13.58 & $5.9-22.6$ & 29.80 & 28.41 & 1.39 & 3.55 & 39.25 & 40.20 & 0.95 & 10.67 & 78.95 \\
\hline
\end{tabular}


Table.3 Correlation matrix showing genotypic and phenotypic correlations among various characters

\begin{tabular}{|c|c|c|c|c|c|c|c|c|c|c|c|c|c|c|c|c|c|c|}
\hline Characters & & $\begin{array}{c}\text { Nodule } \\
\text { dry } \\
\text { wt.(mg) }\end{array}$ & $\begin{array}{c}\text { Pri } \\
\operatorname{mar}_{\mathrm{y}} \\
\text { root } \\
\text { no. }\end{array}$ & $\begin{array}{l}\text { Root } \\
\text { length } \\
\text { (c } \\
\text { m) }\end{array}$ & $\begin{array}{l}\text { Root } \\
\text { dry } \\
\text { weigh } \\
\text { t } \\
(\mathrm{g})\end{array}$ & $\begin{array}{l}\text { Shoot } \\
\text { length } \\
(\mathrm{cm})\end{array}$ & $\begin{array}{l}\text { Shoot } \\
\text { dry } \\
\text { biomass } \\
\text { (g) }\end{array}$ & $\begin{array}{c}\text { Days } \\
\text { to } \\
50 \% \text { fl } \\
\text { oweri } \\
\text { ng }\end{array}$ & $\begin{array}{c}\text { Days } \\
\text { to } \\
\text { maturi } \\
\text { ty }\end{array}$ & $\begin{array}{l}\text { Plant } \\
\text { height } \\
(\mathrm{cm})\end{array}$ & $\begin{array}{l}\text { Number } \\
\text { of } \\
\text { primary } \\
\text { branche } \\
\text { s }\end{array}$ & $\begin{array}{l}\text { Number } \\
\text { of } \\
\text { secondary } \\
\text { branches }\end{array}$ & $\begin{array}{c}\text { No of } \\
\text { pod/pl } \\
\text { ant }\end{array}$ & $\begin{array}{c}100 \text { Pod } \\
\text { wt.(g) }\end{array}$ & $\begin{array}{c}\text { No of } \\
\text { seeds/ } \\
\text { pod }\end{array}$ & $\begin{array}{c}100 \\
\text { Seed } \\
\text { wt. }(g)\end{array}$ & $\mathrm{HI}$ & $\begin{array}{c}\text { Seed } \\
\text { yield/pl } \\
\text { ant(g) }\end{array}$ \\
\hline \multirow{2}{*}{ Nodule no } & $\mathrm{G}$ & $0.582 * *$ & 0.270 & -0.008 & 0.060 & 0.075 & 0.017 & -0.541 & $-0.365 *$ & 0.166 & $0.457 * *$ & -0.099 & $0.366^{*}$ & 0.042 & -0.090 & 0.259 & 0.361 & 0.296 \\
\hline & $\mathrm{P}$ & $0.574 * *$ & 0.252 & -0.037 & 0.001 & 0.052 & -0.078 & -0.590 & $-0.367 *$ & 0.164 & $0.404 *$ & -0.176 & $0.366^{*}$ & 0.020 & -0.094 & 0.258 & 0.351 & 0.284 \\
\hline Dry Nodule & $\mathrm{G}$ & & 0.200 & 0.286 & 0.320 & 0.092 & 0.314 & -0.442 & -0.160 & -0.017 & $0.623 * *$ & 0.255 & $0.736 * *$ & $0.548 * *$ & -0.120 & 0.262 & \multicolumn{2}{|c|}{$0.779 * * 0.688 * *$} \\
\hline Weight(mg) & $\mathrm{P}$ & & 0.184 & 0.271 & 0.296 & 0.076 & 0.302 & -0.412 & -0.165 & -0.053 & $0.516 * *$ & 0.230 & $0.719 * *$ & $0.528 * *$ & -0.122 & 0.260 & \multicolumn{2}{|c|}{$0.760 * * 0.658 * *$} \\
\hline Primary root & $\mathrm{G}$ & & & $0.501 * *$ & $0.692 * *$ & $0.780 * *$ & $0.554 * *$ & 0.137 & $0.607 * *$ & $0.416^{*}$ & 0.144 & $0.487 * *$ & 0.049 & -0.054 & 0.010 & $0.731 * *$ & 0.034 & 0.006 \\
\hline no. & $\mathrm{P}$ & & & $0.490 * *$ & $0.688 * *$ & $0.778 * *$ & $0.533 * *$ & 0.122 & $0.576 * *$ & $0.353 *$ & 0.139 & $0.425^{*}$ & 0.037 & -0.072 & -0.002 & $0.723 * *$ & 0.030 & 0.005 \\
\hline Root length & $\mathrm{G}$ & & & & $0.648 * *$ & $0.378^{*}$ & $0.779 * *$ & 0.080 & $0.470 * *$ & 0.197 & 0.070 & $0.355^{*}$ & 0.044 & 0.077 & -0.026 & 0.278 & 0.137 & 0.048 \\
\hline$(\mathrm{cm})$ & $\mathrm{P}$ & & & & $0.646^{* *}$ & $0.366^{*}$ & $0.724 * *$ & 0.073 & $0.442 *$ & 0.180 & 0.047 & 0.321 & 0.026 & 0.061 & -0.030 & 0.277 & 0.133 & 0.047 \\
\hline Root dry & $\mathrm{G}$ & & & & & $0.580 * *$ & $0.888 * *$ & $0.360 *$ & $0.459 * *$ & 0.215 & 0.058 & $0.386^{*}$ & 0.187 & 0.185 & -0.280 & $0.706 * *$ & 0.290 & 0.221 \\
\hline weight (g) & $\mathrm{P}$ & & & & & $0.578 * *$ & $0.860 * *$ & 0.314 & $0.389 *$ & 0.205 & -0.016 & $0.359 *$ & 0.175 & 0.172 & -0.317 & $0.664 * *$ & 0.261 & 0.201 \\
\hline Shoot length & G & & & & & & $0.544 * *$ & -0.025 & $0.401 *$ & 0.042 & -0.037 & 0.226 & -0.242 & -0.243 & 0.073 & $0.555^{* *}$ & -0.272 & -0.239 \\
\hline$(\mathrm{cm})$ & $\mathrm{P}$ & & & & & & $0.518 * *$ & -0.026 & $0.381 *$ & 0.016 & -0.088 & 0.213 & -0.264 & -0.275 & 0.059 & $0.550 * *$ & -0.275 & -0.250 \\
\hline Shoot dry & G & & & & & & & 0.236 & 0.306 & 0.113 & 0.006 & $0.477 * *$ & 0.127 & 0.160 & 0.008 & $0.386 *$ & 0.228 & 0.099 \\
\hline biomass (g) & $\mathrm{P}$ & & & & & & & 0.183 & 0.230 & 0.010 & -0.142 & $0.455^{* *}$ & 0.109 & 0.151 & -0.044 & $0.334 *$ & 0.180 & 0.098 \\
\hline Days to $50 \%$ & $\mathrm{G}$ & & & & & & & & $0.490 * *$ & 0.268 & -0.229 & 0.276 & -0.182 & -0.022 & -0.291 & 0.250 & -0.156 & -0.167 \\
\hline flowering & $\mathrm{P}$ & & & & & & & & $0.439 *$ & 0.221 & -0.349 & 0.246 & -0.223 & -0.062 & -0.293 & 0.236 & -0.170 & -0.173 \\
\hline Days to & G & & & & & & & & & $0.690 * *$ & -0.107 & $0.416^{*}$ & -0.077 & 0.108 & -0.140 & $0.510 * *$ & -0.158 & -0.039 \\
\hline Maturity & $\mathrm{P}$ & & & & & & & & & $0.535 * *$ & -0.112 & 0.304 & -0.078 & 0.107 & -0.190 & $0.498 * *$ & -0.165 & -0.045 \\
\hline Plant height & G & & & & & & & & & & -0.012 & 0.081 & 0.197 & 0.246 & -0.291 & $0.572 * *$ & 0.067 & 0.164 \\
\hline$(\mathrm{cm})$ & $\mathrm{P}$ & & & & & & & & & & -0.073 & 0.076 & 0.157 & 0.203 & -0.374 & $0.463 * *$ & 0.056 & 0.147 \\
\hline Number of Primary & $\mathrm{G}$ & & & & & & & & & & & 0.045 & $0.416 *$ & 0.128 & -0.259 & 0.335 & $0.525 * *$ & 0.271 \\
\hline branches & $\mathrm{P}$ & & & & & & & & & & & 0.035 & 0.345 & 0.122 & -0.399 & 0.286 & $0.400^{*}$ & 0.254 \\
\hline Number of secondary & $\mathrm{G}$ & & & & & & & & & & & & 0.236 & 0.157 & $0.424 *$ & 0.169 & 0.272 & 0.201 \\
\hline branches & $\mathrm{P}$ & & & & & & & & & & & & 0.185 & 0.129 & 0.180 & 0.132 & 0.233 & 0.146 \\
\hline No. of & $\mathrm{G}$ & & & & & & & & & & & & & $0.900 * *$ & 0.050 & 0.095 & $0.962 * *$ & $0.968 * *$ \\
\hline Pods/Plant & $\mathrm{P}$ & & & & & & & & & & & & & $0.884 * *$ & 0.022 & 0.084 & $0.921 * *$ & $0.934 * *$ \\
\hline 100 Pod & $\mathrm{G}$ & & & & & & & & & & & & & & 0.050 & 0.001 & $0.808 * *$ & $0.882 * *$ \\
\hline Weight & $\mathrm{P}$ & & & & & & & & & & & & & & 0.047 & -0.018 & $0.755^{* *}$ & $0.826 * *$ \\
\hline No of Seeds/Pod & $\mathrm{G}$ & & & & & & & & & & & & & & & $-0.561-$ & $-0.067-$ & $0.029-$ \\
\hline & $\mathrm{P}$ & & & & & & & & & & & & & & & 0.666 & 0.069 & 0.028 \\
\hline 100 Seed & G & & & & & & & & & & & & & & & & 0.137 & 0.090 \\
\hline Weight & $\mathrm{P}$ & & & & & & & & & & & & & & & & 0.135 & 0.082 \\
\hline HI & $\mathrm{G}$ & & & & & & & & & & & & & & & & & $0.939 * *$ \\
\hline & $\mathrm{P}$ & & & & & & & & & & & & & & & & & $0.906 * *$ \\
\hline
\end{tabular}


Table.4 Path analysis of yield attributing characters with seed yield

\begin{tabular}{|c|c|c|c|c|c|c|c|c|c|c|c|c|c|c|c|c|c|c|}
\hline Characters & Nodule no & $\begin{array}{l}\text { Nodule dry } \\
\text { wt.(mg) }\end{array}$ & $\begin{array}{l}\text { Primary } \\
\text { root no. }\end{array}$ & $\begin{array}{l}\text { Root } \\
\text { length }(\mathrm{cm})\end{array}$ & $\begin{array}{l}\text { Root dry } \\
\text { wt.(g) }\end{array}$ & $\begin{array}{l}\text { Shoot } \\
\text { length }(\mathrm{cm})\end{array}$ & $\begin{array}{l}\text { Shoot dry } \\
\text { biomass(g) }\end{array}$ & $\begin{array}{l}50 \% \\
\text { flowering }\end{array}$ & $\begin{array}{l}\text { Days to } \\
\text { maturity }\end{array}$ & $\begin{array}{l}\text { Plant } \\
\text { height }(\mathrm{cm})\end{array}$ & Primary & $\begin{array}{l}\text { Secondary } \\
\text { branch }\end{array}$ & $\begin{array}{l}\text { No of } \\
\text { pod/plant }\end{array}$ & $\begin{array}{l}100 \text { Pod } \\
\text { wt. (g) }\end{array}$ & $\begin{array}{l}\text { No of } \\
\text { seed/pod }\end{array}$ & \begin{tabular}{|l}
100 \\
SEED \\
wt.(g)
\end{tabular} & HI & $\begin{array}{l}\text { Seed } \\
\text { yield/plant(g }\end{array}$ \\
\hline Nodule no & -0.037 & 0.190 & -0.154 & -0.021 & 0.002 & 0.019 & 0.150 & -0.060 & 0.049 & 0.015 & -0.153 & -0.009 & 0.127 & -0.001 & -0.038 & -0.005 & 0.221 & 0.296 \\
\hline $\begin{array}{l}\text { Nodule dry } \\
\text { Weight(mg) }\end{array}$ & -0.021 & 0.331 & -0.112 & 0.151 & 0.466 & 0.028 & -0.581 & -0.045 & 0.022 & -0.005 & -0.208 & 0.014 & 0.255 & -0.027 & -0.051 & -0.005 & 0.477 & $0.688^{* * *}$ \\
\hline Primary root no. & -0.009 & 0.061 & -0.611 & 0.273 & 1.091 & 0.290 & -1.065 & 0.014 & -0.082 & 0.038 & -0.046 & 0.026 & 0.013 & 0.004 & 0.004 & -0.014 & 0.021 & 0.006 \\
\hline Root length(cm) & 0.001 & 0.090 & -0.300 & 0.557 & 1.018 & 0.136 & -1.496 & 0.008 & -0.063 & 0.018 & -0.016 & 0.019 & 0.009 & -0.003 & -0.011 & -0.005 & 0.084 & 0.048 \\
\hline Root dry weight(g) & 0.000 & 0.098 & -0.423 & 0.360 & 1.576 & 0.215 & -1.705 & 0.036 & -0.062 & 0.019 & 0.006 & 0.021 & 0.061 & -0.009 & -0.134 & -0.014 & 0.177 & 0.221 \\
\hline Shoot length(cm) & -0.002 & 0.025 & -0.476 & 0.204 & 0.911 & 0.372 & -1.045 & -0.002 & -0.054 & 0.001 & 0.030 & 0.012 & -0.092 & 0.014 & 0.031 & -0.011 & -0.168 & -0.239 \\
\hline Shoot dry biomass(g) & 0.003 & 0.100 & -0.339 & 0.434 & 1.399 & 0.203 & -1.921 & 0.024 & -0.041 & -0.001 & 0.048 & 0.025 & 0.038 & -0.007 & 0.003 & -0.007 & 0.140 & 0.099 \\
\hline $50 \%$ Flowering & 0.022 & -0.146 & -0.084 & 0.045 & 0.568 & -0.009 & -0.453 & 0.101 & -0.066 & 0.025 & 0.117 & 0.015 & -0.078 & 0.003 & -0.123 & -0.005 & -0.104 & -0.167 \\
\hline Days to Maturity & 0.014 & -0.053 & -0.371 & 0.262 & 0.724 & 0.149 & -0.588 & 0.049 & -0.135 & 0.063 & 0.038 & 0.022 & -0.027 & -0.005 & -0.080 & -0.010 & -0.097 & -0.039 \\
\hline Plant Height & -0.006 & -0.017 & -0.254 & 0.110 & 0.324 & 0.006 & 0.020 & 0.027 & -0.093 & 0.092 & 0.024 & 0.004 & 0.068 & -0.012 & -0.158 & -0.011 & 0.041 & 0.164 \\
\hline Primary Branch & -0.017 & 0.206 & -0.085 & 0.026 & -0.026 & -0.033 & 0.274 & -0.035 & 0.015 & -0.007 & -0.334 & 0.002 & 0.144 & -0.006 & -0.169 & -0.007 & 0.321 & 0.271 \\
\hline Secondary Branch & 0.007 & 0.084 & -0.298 & 0.198 & 0.609 & 0.084 & -0.917 & 0.028 & -0.056 & 0.007 & -0.015 & 0.053 & 0.082 & -0.008 & 0.179 & -0.003 & 0.166 & 0.201 \\
\hline No. of Pods/Plant & -0.014 & 0.243 & -0.023 & 0.015 & 0.276 & -0.098 & -0.210 & -0.023 & 0.010 & 0.018 & -0.139 & 0.013 & 0.347 & -0.045 & 0.009 & -0.002 & 0.588 & $0.968^{* *}$ \\
\hline 100 Pod Wt. & -0.001 & 0.181 & 0.044 & 0.034 & 0.271 & -0.102 & -0.290 & -0.006 & -0.015 & 0.023 & -0.043 & 0.008 & 0.312 & -0.050 & 0.020 & 0.000 & 0.494 & $0.882 * *$ \\
\hline No of Seed/Pod & 0.003 & -0.040 & -0.006 & -0.014 & -0.500 & 0.027 & -0.016 & -0.029 & 0.026 & -0.034 & 0.133 & 0.023 & 0.008 & -0.002 & 0.423 & 0.013 & -0.041 & 0.029 \\
\hline 100 Seed Wt. & -0.010 & 0.087 & -0.447 & 0.154 & 1.113 & 0.207 & -0.741 & 0.025 & -0.069 & 0.053 & -0.112 & 0.009 & 0.029 & 0.001 & -0.282 & -0.019 & 0.084 & 0.090 \\
\hline HI & -0.013 & 0.258 & -0.021 & 0.076 & 0.457 & -0.103 & -0.439 & -0.017 & 0.021 & 0.006 & -0.175 & 0.014 & 0.334 & -0.040 & -0.028 & -0.003 & 0.612 & 0.939 ** \\
\hline
\end{tabular}

Residual effect $=0.2890518$ 
The desi type viz. BG 256, Pusa 362, PDG 3, GNG 146 and CSJD 884 had more nodule dry weight per plant and seed yield per plant. Among kabuli type HK 1 and HK 2 had high nodule weight but low seed yield per plant was observed as compare to L 550 and the reason behind this may be due to presence of more ineffective nodules in HK 1 and HK 2. In case of majority of varieties, microsperma races (desi type) had better nodulating behaviour including nodule number per plant, nodule dry weight per plant and better economic yield potential in contrast to macrosperma races (kabuli type). One of the kabuli variety L550 performed better than few of the desi varietes i.e. HC 3, BGM 408, GPF 2 and Anuradha. Considering most of the characters (both underground and above ground) the variety BG 256 and Pusa 362 appeared to be very good performer at New Alluvial Zone.

\section{Acknowledgement}

The authors gratefully acknowledge the help and cooperation received from Dr. H.K. Sarker, Ex-Professor and Head, Department of Genetics and Plant Breeding, Bidhan Chandra Krishi Viswavidyalaya, Mohanpur741252, Nadia, West Bengal, India

\section{References}

Bender, D. A., and Bender, A. E. 2005. A Dictionary of food and nutrition. New York: Oxford University Press. ISBN 0198609612.

Borate, V. V., Dalvi, V. V. and Jadhav, B. B. 2010. Estimates of genetic variability and heritability in chickpea. Journal of Maharashtra Agricultural Universities. 35(1): 47-49.

FAO, 2013. FAOSTAT. Chickpea harvested area, 2013. Food and Agriculture Organization data base of the United Nations.
[Http://faostat.fao.org/site/567/Desktop

Default.aspx? PageID=567\#ancor].

Kantar, F., Hafeez, F. Y., Shivakumar, B. G., Sundaram, S. P., Tejera, N. A., Aslam, A., Bano, A. and Raja, P. 2007. Chickpea Rhizobium management and nitrogen fixation. Chickpea Breeding Management. 179-192.

Khan, H., Ahmad, S. Q., Ahmad, F., Khan, M. S. and Iqbal, N. 2006. Genetic variability and correlations among quantitative traits in gram. Sarhad Journal of Agriculture. 22: 55-59.

Khattak, S., Khan, D. F., Shah, S. H., Madani, M. S. and Khan, T. 2006. Role of Rhizobial inoculation in the production of chickpea crop. Soil and Environment. 25(2): 143-145.

Mansfeld, 2008. Mansfeld's World Database of Agricultural and Horticultural Crops: Cicer arietinum subsp. Aritinum [Http://mansfeld.ipkgatersleben.de/pls/h tmldb_pgrc/f?p=185:46:774070393638 961::NO::module,source,taxid,akzname :mf,volksnam,30808,Cicer\%20arietinu m\%20subsp.\%20arietinum].

Moreno, M. T., and Cubero, J. I. 1978. Variation in Cicer arietinum L. Euphytica. 27: 465-468.

Patel, J. D., Desai, N. C., Intwala, C. G. and Kodappully, V. C. 2008. Genetic variability, correlation and path analysis in moth bean. Journal of Food Legumes. 21(3): 158-160.

Ramgiry, S. R., 1997. Genetic variability in Indian germplasm of faba bean (Vicia faba L.) in relation to yield and nodulation characters. Advances in Plant Sciences. 10(1): 173-176.

Rana, O. P. S., Maherchandani, N., Singh, K. P. 1995. Studies on nodulation and its association with seed yield and its components in chickpea (Cicer arietinum L.). Crop Research Hisar. 9(2): 324-329.

Romdhane, S. B., Aouani, M. E., Trabelsi, 
M., DeLajudie, P. and Mhamdi, R. 2008. Selection of High Nitrogen Fixing Rhizobia Nodulating Chickpea (Cicer arietinum) for Semi-Arid Tunisia. Journal of Agronomy and Crop Science. 194 (6): $413-420$.
Singh, I., Phul, P. S., Singh, T. P., Gupta, R. P. and Sharma, S. R. (1995). Genetic variability and response of soybean genotypes to Bradyrhizobium japonicum inoculation. Journal of Research, Punjab Agricultural University. 32(3): 245- 252.

\section{How to cite this article:}

Priyadarsini, L., Prabhat K. Singh, C. Chatterjee, R. Sadhukhan and Biswas, T. 2017. Estimation of Genetic Variability of Nodulation Characters and their Association with Different Agromorphic Characters and Yield in Chickpea (C. arietinum L.). Int.J.Curr.Microbiol.App.Sci. 6(9): 1928-1935. doi: https://doi.org/10.20546/ijcmas.2017.609.236 